\section{Effects of a branched-chain amino acid drink}

\section{in mania}

\author{
A. SCARNÀ, H. J. GIJSMAN, S. F. B. MCTAVISH, C. J. HARMER, P. J. COWEN \\ and G. M. GOODWIN
}

\begin{abstract}
Background Administration of a complex tyrosine-free amino acid drink acutely decreases manic symptoms. Although a nutrient-based approach to illness management is attractive, complex amino acid drinks are too unpalatable for repeated administration.
\end{abstract}

Aims To assess whether a simple, branched-chain amino acid (BCAA) drink diminishes manic symptoms acutely and following repeated administration.

Method Twenty-five patients with mania were randomly and blindly allocated to treatment with BCAA $(60 \mathrm{~g})$ or placebo daily for 7 days.

Results Relative to placebo, the BCAA drink lowered mania ratings acutely over the first $6 \mathrm{~h}$ of treatment. In protocol completers there was a persistent advantage to the BCAA group I week after the end of treatment.

Conclusions Anutritional intervention that decreases tyrosine availability to the brain acutely ameliorates manic symptoms. Further studies are required to assess whether this approach has longerterm efficacy.

\section{Declaration of interest This study} was funded by the Wellcome Trust; P.J.C. is a Medical Research Council (MRC) Clinical Scientist, S.F.B.M. is an MRC Clinician Scientist Fellow and C.J.H. is an MRC Training Fellow.
In experimental studies in animals, acute administration of an amino acid mixture lacking the catecholamine precursors tyrosine and phenylalanine (tyrosine-free) decreases the availability of plasma tyrosine to the brain. This diminishes catecholamine synthesis and attenuates the dopaminereleasing properties of amphetamine (McTavish et al, 1999). Similarly, in humans, administration of a tyrosine-free amino acid load attenuates both subjective and objective effects of methamphetamine in healthy volunteers (McTavish et al, 2001). The ability of amphetamine to release dopamine has been linked to its psychostimulant effects, making it a plausible model of mania (Jacobs \& Silverstone, 1986). A preliminary study in people with mania showed that a single dose of the tyrosine-free amino acid load acutely decreased symptom severity on an observerrated clinical scale over $6 \mathrm{~h}$ (McTavish et al, 2001). However, the amino acid drink used was unpalatable and unsuitable for the repeated administration required in the treatment of clinical disorders.

In our study we used a simpler amino acid mixture containing only three branched-chain amino acids (BCAAs): leucine, isoleucine and valine. These amino acids compete with phenylalanine and tyrosine for brain entry, and studies in healthy volunteers indicate that BCAAs produce cognitive and endocrine effects consistent with attenuation of central dopamine neurotransmission (Gijsman et al, 2002). In addition, the BCAA drink is palatable, safe and well tolerated during longer-term ingestion (Richardson et al, 1999). The effect of the BCAA drink on manic symptoms was assessed both acutely and after repeated administration.

\section{METHOD}

\section{General study design}

We compared a drink containing BCAAs with a placebo drink for the treatment of acute mania. This intervention was added to treatment as usual, lasted for 7 days and was followed by a 7-day follow-up period. The protocol was approved by the local ethics committee and all participants gave informed consent before randomisation.

\section{Participants}

Patients participating in the study fulfilled DSM-IV criteria for bipolar I disorder with a current manic episode (American Psychiatric Association, 1994) and were assessed to have a score of 20 or more on the Young Mania Rating Scale (YMRS; Young et al, 1978). These patients were recruited from five acute wards within the Oxfordshire Mental Healthcare National Health Service Trust. The main exclusion criteria were serious physical illness and the inability to give informed consent in the opinion of either the ward consultant or the investigators. We also excluded patients who were taking sodium valproate, because both valproate and BCAA may have hepatotoxic effects and we did not wish to risk a possible interaction.

\section{Intervention}

Each patient received a drink containing $60 \mathrm{~g}$ amino acid or a seemingly identical placebo. The experimental mixture consisted of valine, isoleucine and leucine in the ratio 3:3:4. The control drink contained fatty flakes to simulate the texture of the experimental mixture. Both drinks also contained flavouring, peanut oil and water, as described elsewhere (Gijsman et al, 2002; Scarnà et al, 2002). All drinks were prepared and administered by a research nurse not involved in the further execution of the study. Prescribed medication and food were not withheld during the study period. The drink was administered once daily in the morning.

\section{Objectives}

To assess the tolerability and effect of a drink containing BCAAs compared with placebo on the symptoms of acute mania, specifically over $6 \mathrm{~h}$ after the first drink as well as during and after a treatment period of 7 days.

\section{Outcomes}

The YMRS was used as the sample selection tool. Our outcome measure was the Beigel Manic State Rating Scale, which is sensitive to clinical changes over short 
periods and reflects a wider spectrum of symptoms (Beigel \& Murphy, 1971; McTavish et al, 2001). Participants were assessed immediately before administration of the first drink and then hourly for $6 \mathrm{~h}$. Subsequent ratings were carried out on days 4 and 8 , and 1 week after the end of treatment on day 15, after a 1-h observation period and interview.

\section{Sample size}

A single dose of a tyrosine-free amino acid drink reduced manic symptoms by $35 \%$ in a similar study with group size of 10 (McTavish et al, 2001). For the study described here we aimed at a similar effect after a single dose and therefore required a group size of at least 10 . For the 15-day follow-up we aimed at 10 completers in each group.

\section{Randomisation}

An independent investigator generated a randomisation sequence on a computer. Randomisation was calculated in blocks of four. Allocation was concealed by using numbered and weight-balanced boxes containing all the ingredients for the treatment of one patient. The independent supplier of the ingredients prepared these boxes. Patients included in the study were given a sequential number and the corresponding box was opened by the independent research nurse when preparing the first drink.

\section{Masking}

Participants and investigators were masked to group assignment. The success of masking was not separately assessed.

\section{Statistical methods}

For the single dose study, Beigel scores were analysed as change from baseline and compared using a repeated-measures analysis of variance (ANOVA) with 'time' (time of rating) as the within-subject factor and 'drink' (BCAA or placebo) as the between-subject factor. For the 15-day follow-up the Beigel scores were also analysed as change from baseline. Repeated-measures ANOVAs were conducted with 'day' (day of rating) as the main within-subject factor and 'drink' (BCAA or placebo) as the betweensubject factor. For all ANOVAs, uncorrected degrees of freedom are reported for reasons of clarity, but Huynh-Feldt correction was used. All $t$-tests are reported with equal variances not assumed.

\section{RESULTS}

\section{Participant flow}

Recruitment took place between January and December 2001. Forty-nine patients considered suitable were screened. Of these, 13 patients scored below 20 on the YMRS, 1 was unable to provide informed consent, 3 were taking sodium valproate and 6 refused to participate. One further patient was unsuitable for other clinical reasons. The remaining 25 patients were randomised. Thirteen participants received the BCAA drink and 12 were given the placebo. Seven participants discontinued the allocated treatment: 4 after day 1, and 3 after day 2. Two participants in the placebo group were withdrawn from the study for clinical reasons, unrelated to the drink. Of the five who withdrew from the BCAA group, one complained of sleepiness and another of nausea, both possibly related to the drink. Another participant cited concern about protein as a reason for not completing the study, and a fourth expressed some paranoid ideas regarding contents of the drink. The fifth participant did not give a reason for discontinuing. Eighteen participants (8 BCAA, 10 placebo) completed the treatment protocol. There was no drop-out from follow-up and all 25 participants were assessed up to day 15 .

\section{Baseline data}

Patients were aged 19-62 years (mean 41 years). Three patients were experiencing a first episode of mania; the others had a mean of 2 previous hospital admissions for manic illness (range 1 to 4 ). The medication used for each patient was left to the discretion of the psychiatrist. Twenty-two patients were receiving antipsychotic medication during the study. Nine of these participants were also receiving treatment with a mood stabiliser: 5 were receiving carbamazepine, and 4 were receiving lithium. Mood stabiliser monotherapy had not failed in this group of patients; rather, the standard treatment for most of the consultant psychiatrists was an antipsychotic plus a benzodiazepine. Of the remaining 3 participants, 1 was taking lithium only, 1 carbamazepine only and 1 both drugs combined.

Fifteen of the participants received intermittent treatment with benzodiazepines. In the placebo group, 5 patients were taking lorazepam and 1 patient was taking zopiclone. One patient had taken lorazepam on one occasion before being changed to zopiclone. In the BCAA group, 5 patients were taking lorazepam, 2 clonazepam and 1 diazepam. When converted to diazepam equivalents, there was little difference between the average amounts taken at the start of the study $(5.4 \mathrm{mg}$ for the placebo group, $6.5 \mathrm{mg}$ for the BCAA group) and this did not differ significantly throughout the duration of the study.

The mean score on the YMRS was 29 (range 21-42), within the range of that seen in drug treatment studies of mania (Tohen et al, 2000). The two groups (BCAA $v$. placebo) were well matched for baseline severity of manic symptoms on the YMRS: 29.3 (s.d. 5.2) v. 29.2 (s.d. 7.2); age: 41.3 (s.d. 12.4) v. 40.3 (s.d. 12.4) years; and gender: $6 / 13$ v. 4/12 female.

\section{Numbers analysed}

All 25 participants received the allocated intervention on day 1 of the study. These participants completed all follow-up measurements and were included in the intention-to-treat analysis. Additionally, we analysed data from the 18 participants who received the full 7 days of treatment.

\section{Outcomes}

Administration of the BCAA drink on day 1 significantly reduced clinical ratings of mania as measured by change from baseline on the Beigel scale relative to the placebo drink (Fig. 1). A two-way ANOVA showed

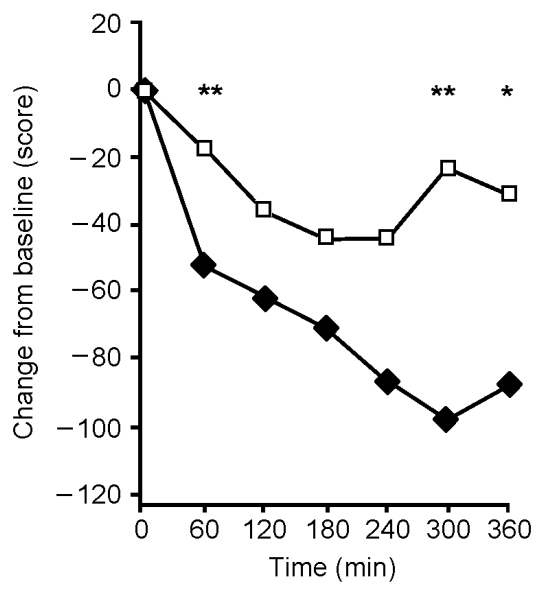

Fig. I Mean scores on the Beigel Manic State Rating Scale (measured as change from baseline) following a branched-chain amino acid (BCAA) drink $(\diamond)(n=13)$ and placebo $(\square)(n=12)$ given at time 0 . Beigel ratings were significantly lower after the BCAA drink $(F=2.84, P=0.02) . * P<0.05$, $* * P<0.0$. 
significant effects of time $(F=10.21$, d.f. $=6,138, \quad P<0.01)$ and of drink $(F=9.81$, d.f. $=1,23, P=0.01)$ and a significant interaction $(F=2.84$, d.f. $=6,138$, $P=0.02)$. Post hoc testing showed significant differences between BCAA and control drinks at three time points (Fig. 1).

Over the subsequent 2 weeks both treatment groups showed a fall in clinical ratings of mania on the intention-to-treat analysis (Fig. 2). The ANOVA showed a significant effect of day $(F=13.81$, d.f. $=3,69, P<0.01)$ but no significant effect of drink $(F=1.67$, d.f. $=1,23, P=0.21)$ and no significant interaction $(F=1.35$, d.f. $=3,69, P=0.27$ ). The analysis of treatment completers showed significant effects of day $(F=10.62$, d.f. $=3,48, P<0.01)$ and of drink $(F=41.52$, d.f. $=1,16, P<0.01)$ and a significant interaction $(F=10.16$, d.f. $=3,48, P<0.01)$. Pairwise comparisons showed only a trend in difference between groups on day $4\left(t_{1,16}=1.84, P=0.09\right)$, no difference on day $8\left(t_{1,16}=0.89, P=0.39\right)$, and a significant difference between groups on day $15\left(t_{1,16}=7.5, P<0.01\right)$. The placebo effect diminished during the week of treatment, whereas the BCAA group continued to improve (Fig. 3).

\section{Adverse events}

The BCAA drink was generally well tolerated over the period of the study and the majority of the patients had no problem in swallowing it. The only substantial adverse effects were reported by the two participants in the BCAA group who discontinued the treatment because of nausea or tiredness.

\section{DISCUSSION}

\section{Acute effects of branched-chain amino acids on manic symptoms}

The finding that acute administration of the BCAA drink decreased ratings of mania is consistent with our earlier observation with a more-complex tyrosine-free amino acid mixture (McTavish et al, 2001) and suggests that BCAA administration might be a viable way of attenuating dopaminergic overactivity in pathological states. It is of additional interest that in this study the control drink was a true placebo. Our original study employed a 'balanced' drink containing substantial doses of tyrosine, making it possible that the difference in effects simply reflected a contrast between a high-tyrosine and low-tyrosine state. For example, a recent study in volunteers suggests that a tyrosine-containing drink can increase alertness (Grevet et al, 2002).

In fact, it is possible that the BCAA drink is less effective than the full tyrosine-free amino acid mixture in acutely reducing the symptoms of mania. In the current study the people treated with BCAA showed a decrease in ratings on the Beigel scale that was about $20 \%$ greater than that of the placebo group. This is somewhat less than the $35 \%$ reduction in symptoms seen in our previous study (McTavish et al, 2001). One possible explanation for this lesser effect is that the BCAA drink used by McTavish et al might have been less active at lowering brain tyrosine availability, because the full tyrosine-free amino acid mixture lowers plasma tyrosine concentrations as well as decreasing its brain entry by competition at the blood-brain barrier. In contrast, the BCAA drink used in the current study would be expected to lower brain tyrosine by competition only (Gijsman et al, 2002). Another possibility is that the tyrosine-containing control amino acid mixture used in our previous study could have caused some activation (Grevet et al, 2002), lowering the placebo response and inflating the apparent effect of the tyrosine-free mixture.

\section{Longer-term effects}

Both patient groups showed an amelioration of symptoms over the 2-week period, with most measures yielding significant effects of day, but not of drink. This is not surprising since routine treatment was not controlled and patients continued to receive both routine and 'as required' medication on the usual basis of perceived clinical need. An intention-to-treat analysis failed to differentiate placebo from the BCAA treatment, but despite the power limitations of such a small study, there were hints of additional benefit in the BCAA group. For example, 4 of the 8 BCAA patients were discharged before day 15 , compared with only 2 from the 10 in the placebo group.

When the results from the study completers (participants who accepted the full treatment protocol) were assessed, there appeared to be a waning of the symptomatic improvement in the placebo group after treatment cessation, but a continued improvement in the BCAA group participants

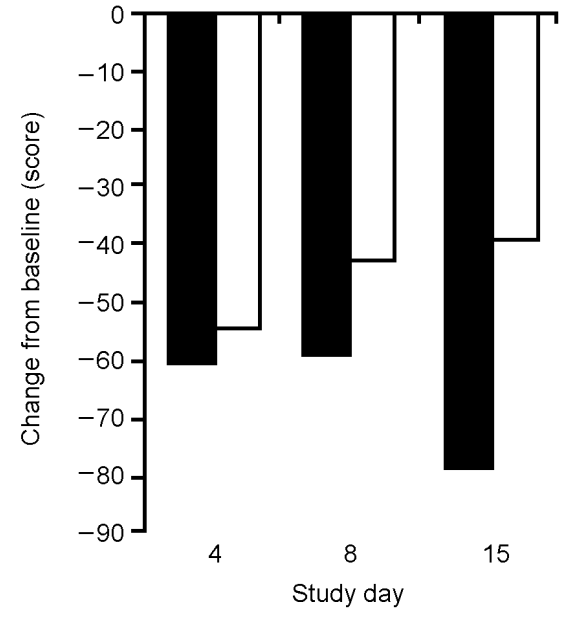

Fig. 2 Mean scores on the Beigel Manic State Rating Scale (measured as change from baseline) after repeated administration of a branched-chain amino acid drink $(\square)(n=13)$ and placebo $(\square)(n=12)$ for I week in intention-to-treat analysis. The effects of the two treatments do not differ significantly $(F=1.67, P=0.2 \mathrm{I})$.

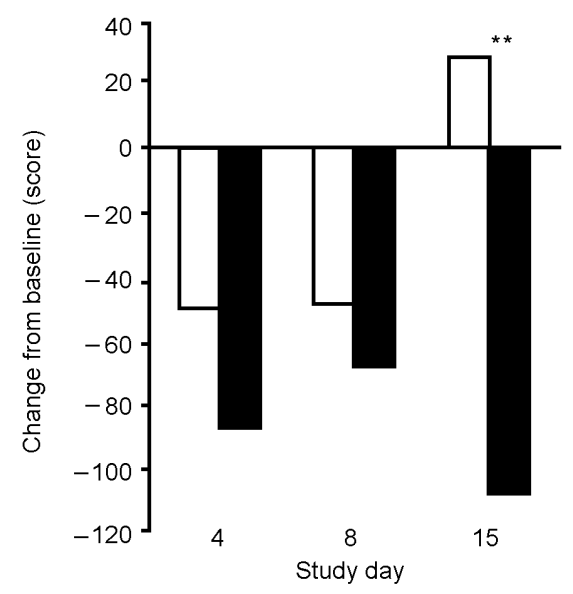

Fig. 3 Mean scores on the Beigel Manic State Rating Scale (measured as change from baseline) after repeated administration of a branched-chain amino acid drink $(\square)(n=8)$ and placebo $(\square)(n=10)$ for I week in completer analysis. The treatment effects are significantly different $(F=10.16, P<0.01)$ on day 15 . $* * P<0.01$.

which was statistically significant on day 15. The reason why the BCAA group should have continued to improve after the end of treatment is not clear. It is possible that an adjunctive effect of the drink for 1 week allowed a more rapid resolution of the manic syndrome even after BCAA treatment ended. In contrast, the placebo improvement seemed to wane as would be expected. 


\section{Mechanism of action of branched-chain amino acids}

Acute BCAA treatment increases plasma prolactin levels and impairs performance on a spatial recognition task, effects consistent with attenuation of dopamine neurotransmission (Gijsman et al, 2002). In addition, using positron emission tomography we have found that a full tyrosine-free amino acid mixture increases striatal binding of the dopamine receptor ligand $\left[{ }^{11} \mathrm{C}\right]$ raclopride in healthy volunteers, an effect consistent with lowered presynaptic dopamine release (Montgomery et al, 2002).

Although we believe that there is now strong evidence that the BCAA mixture impairs dopamine release for the first few hours after administration, we do not know for how long this effect persists and whether it is maintained after repeated treatment. We decided to administer the BCAA drink once daily to increase compliance, but it is possible that more-frequent daily treatment would have produced a better effect. However, administration of BCAAs at high dosage carries potential risks such as increased plasma ammonia concentration hepatotoxicity. In a study in healthy volunteers we monitored plasma ammonia levels after a drink containing $60 \mathrm{~g}$ BCAA, and found that although plasma ammonia concentrations were elevated over $6 \mathrm{~h}$, the average maximum elevation ( $52 \mu \mathrm{mol} / \mathrm{l})$ was below toxic plasma levels ( $>80 \mu \mathrm{mol} / \mathrm{l}$ ) (Scarnà et al, 2001). However, higher doses or longer-term use of BCAA treatment might require liver function test monitoring.

\section{Possible therapeutic utility}

Taken together, our findings suggest that the BCAA drink is a useful and welltolerated tool for modifying dopaminergic function. Although it may be unrealistic to expect such a dietary manipulation to be as potent as an antipsychotic drug, the BCAA drink does appear to achieve an acute reduction in symptoms in patients with mania. Indications of a more persistent effect in speeding symptom resolution will need to be explored in future longerterm trials.

\section{ACKNOWLEDGEMENTS}

We thank R. Hockney and C. Williams for nursing assistance.

\section{CLINICAL IMPLICATIONS}

- A single administration of a palatable branched-chain amino acid (BCAA) drink can acutely diminish the symptoms of acute mania.

- It is possible that repeated BCAA treatment might result in a more rapid resolution of the manic syndrome.

- Dietary manipulations that limit the availability of tyrosine to the brain may be of value in the treatment of psychiatric conditions associated with increased dopaminergic activity.

\section{LIMITATIONS}

- It is uncertain whether a BCAA drink administered without concomitant medication would attenuate manic symptoms.

- The number of patients studied was small and the follow-up period short.

- The effect of BCAA treatment to improve symptom ratings at 15 days was not apparent in the intention-to-treat sample.

A. SCARNA, DPhil, H. J. GIJSMAN, MD, S. F. B. MCTAVISH, MRCPsych, C. J. HARMER, DPhil, P. J. COWEN, FRCPsych, G. M. GOODWIN, FRCPsych, University of Oxford, Warneford Hospital, Oxford, UK

Correspondence: Dr A. Scarnà, Neurosciences Building, University of Oxford, Department of Psychiatry,Warneford Hospital, Oxford OX3 7JX, UK. Tel: 01865 226492; e-mail: anna.scarna@psych.ox.ac.uk

(First received 17 May 2002, final revision 27 September 2002, accepted 10 October 2002)

\section{REFERENCES}

American Psychiatric Association (1994) Diagnostic and Statistical Manual of Mental Disorders (4th edn) (DSM-IV). Washington, DC: APA.

Beigel, A. \& Murphy, D. L. (197I) Assessing clinical characteristics of the manic state. American Journal of Psychiatry, 128, 688-694.

Gijsman, H. J., Scarnà, A., Harmer, C. J., et al (2002) A dose-finding study on the effects of branch chain amino acids on surrogate markers of brain dopamine function. Psychopharmacology, 160, 192-197.

Grevet, E. H., Tietzmann, M. R., Shansis, F. M., et a (2002) Behavioural effects of acute phenylalanine and tyrosine depletion in healthy male volunteers. Journal of Psychopharmacology, 16, 51-55.

Jacobs, D. \& Silverstone, T. (1986)

Dextroamphetamine-induced arousal in human subjects as a model for mania. Psychological Medicine, 16, $323-329$

McTavish, S. F. B. \& Cowen, P. J. (1999) Effect of a tyrosine-free amino acid mixture on regional brain catecholamine synthesis and release. Psychopharmacology, 14I, 182-188.

McPherson, M. H., Harmer, C. J., et al (200I) Antidopaminergic effects of dietary tyrosine depletion in healthy subjects and patients with manic illness. British Journal of Psychiatry, I79, 356-360.
Montgomery, J. A., McTavish, S. F. B., Cowen, P. J., et al (2002) PET measurement of the effect of dietary tyrosine depletion on ["C]raclopride binding. Journal of Psychopharmacology, 16, A42.

Richardson, M. A., Bevans, M. L., Weber, J. B., et al (1999) Branched chain amino acids decrease tardive dyskinesia symptoms. Psychopharmacology, 143 358-364.

Scarnà, A., Gijsman, H. J., Harmer, C. J., et al (200I) Effects of a branch chain amino acid drink supplemented with tryptophan on surrogate markers of brain dopamine function. Journal of Psychopharmacology, I5, Al4.

_, _, , et al (2002) Effects of a branch chain amino acid supplemented with tryptophan on tyrosine availability and plasma prolactin. Psychopharmacology, I59, 222-223.

Tohen, M., Jacobs, T. G., Grundy, S. L., et al (2000) Efficacy of olanzapine in acute bipolar mania. Archives of General Psychiatry, 57, 841-849.

Young, R. C., Biggs, J. T., Ziegler, V. E., et al (1978) A rating scale for mania: reliability, validity and sensitivity. British Journal of Psychiatry, 133, 429-435. 